\title{
Mechanisms for Lasing with Cold Atoms as the Gain Medium
}

\author{
William Guerin, Franck Michaud, and Robin Kaisen* \\ Institut Non Linéaire de Nice, CNRS and Université de Nice Sophia-Antipolis, \\ 1361 route des Lucioles, 06560 Valbonne, France.
}

(Dated: July 18, 2021)

\begin{abstract}
We realize a laser with a cloud of cold rubidium atoms as gain medium, placed in a low-finesse cavity. Three different regimes of laser emission are observed corresponding respectively to Mollow, Raman and Four Wave Mixing mechanisms. We measure an output power of up to $300 \mu \mathrm{W}$ and present the main properties of these different lasers in each regime.

PACS numbers: 33.20.Fb, 37.30.+i, 42.55.Ye, 42.55.Zz, 42.65.Hw
\end{abstract}

Since Letokhov's seminal paper [1], random lasers have received increasing interest. Random lasing occurs when the optical feedback due to multiple scattering in the gain medium itself is sufficiently strong to reach the lasing threshold. In the past decade, it has been observed in a variety of systems (see [2] for a review) but many open questions remain to be investigated, for which better characterized samples would be highly valuable. A cloud of cold atoms could provide a promising alternative medium to study random lasing, allowing for a detailed understanding of the microscopic phenomena and a precise control of essential parameters such as particle density and scattering cross section. These properties have been exploited to study coherent backscattering of light 3] and radiation trapping [4] in large clouds of cold atoms. As many different gain mechanisms have been observed with cold atoms, combining multiple scattering and gain in cold atomic clouds seems a promising path towards the realization of a new random laser. Besides the realization of a random laser, cold atoms might allow to study additional features, such as the transition from superfluorescence [5] to amplified spontaneous emission [6] in a multiple scattering regime. One preliminary step along this research lines is to use a standard cavity to trigger laser oscillation with cold atoms as gain medium. Such a laser may also be an interesting tool for quantum optics, as one can take advantage of the nonlinear response of the atoms to explore nonclassical correlations or obtain squeezing [7].

In this letter, we present the realization of a cold-atom laser, that can rely on three different gain mechanisms, depending on the pumping scheme. By pumping near resonance, Mollow gain 8, 99 is the dominant process and gives rise to a laser oscillation, whose spectrum is large (of the order of the atomic natural linewidth), whereas by pumping further from resonance, Raman gain between Zeeman sublevels 10] gives rise to a weaker, spectrally sharper laser [11]. At last, by using two counterpropagating pump beams, degenerate four-wave mixing (FWM) [12, 13] produces a laser with a power up to $300 \mu \mathrm{W}$. By adjusting the atom-laser detuning or the pump geometry, we can continuously tune the laser from one regime to another.
Our experiment uses a cloud of cold ${ }^{85} \mathrm{Rb}$ atoms confined in a vapor-loaded Magneto-Optical Trap (MOT) produced by six large independent trapping beams, allowing the trapping of up to $10^{10}$ atoms at a density of $10^{10}$ atoms $/ \mathrm{cm}^{3}$, corresponding to an on-resonance optical thickness of about 10. A linear cavity, formed by two mirrors (a coupling-mirror with curvature $R C 1=1 \mathrm{~m}$, reflection coefficient $R 1=0.95$ and plane end mirror with reflection coefficient $R 2 \approx 0.995)$ separated by a distance $L=0.8 \mathrm{~m}$ is placed outside the vacuum chamber, yielding a large round trip loss $\mathcal{L}=32 \%$ with a correspondingly low finesse $\mathcal{F}=16$. The waist of the fundamental mode of the cavity at the MOT location is $w_{\text {cav }} \approx 500 \mu \mathrm{m}$. To add gain to our system, we use either one or two counter-propagating pump beams, denoted F (forward) and B (backward), produced from the same laser with a waist $w_{\text {pump }}=2.6 \mathrm{~mm}$, with linear parallel polarizations and a total available power of $P=80 \mathrm{~mW}$, corresponding to a maximum pump intensity of $I=2 P /\left(\pi w_{\text {pump }}^{2}\right) \approx 750 \mathrm{~mW} / \mathrm{cm}^{2}$. The pump is tuned near the $F=3 \rightarrow F^{\prime}=4$ cycling transition of the $D 2$ line of ${ }^{85} \mathrm{Rb}$ (frequency $\omega_{\mathrm{A}}$, wavelength $\lambda=780 \mathrm{~nm}$, natural linewidth $\Gamma / 2 \pi=5.9 \mathrm{MHz}$ ), with an adjustable detuning $\Delta=\omega_{\mathrm{F}, \mathrm{B}}-\omega_{\mathrm{A}}$ and has an incident angle of $\approx 20^{\circ}$ with the cavity axis. An additional beam $\mathrm{P}$ is used as a local oscillator to monitor the spectrum of the laser or as a weak probe to measure single-pass gain (insets of Figs. 2-4) with a propagation axis making an angle with the cavity axis smaller than $10^{\circ}$. Its frequency $\omega_{\mathrm{P}}$ can be swept around the pump frequency with a detuning $\delta=\omega_{\mathrm{P}}-\omega_{\mathrm{F}, \mathrm{B}}$. Both lasers, pump and probe, are obtained by injection-locking of a common master laser, which allows to resolve narrow spectral features. In our experiments, we load a MOT for $29 \mathrm{~ms}$, and then switch off the trapping beams and magnetic field gradient during $1 \mathrm{~ms}$, when lasing or pump-probe spectroscopy are performed. In order to avoid optical pumping into the dark hyperfine $F=2$ ground state, a repumping laser is kept on all the time. Data acquisitions are the result of an average of typically 1000 cycles.

As in a conventional laser, lasing occurs if gain exceeds losses in the cavity, which can be observed as strong directional light emission from the cavity. As we will discuss 
TABLE I: Different regimes of cold-atom laser versus pump detuning. The polarization of the lasers are either parallel (\|) or orthogonal $(\perp)$ to the polarization of the pump beams.

\begin{tabular}{cccc}
\hline \hline Pump beam(s) & $\Delta<-4 \Gamma$ & $-4 \Gamma<\Delta<+4 \Gamma$ & $\Delta>+4 \Gamma$ \\
\hline $\mathrm{F}$ & Raman $(\perp)$ & Mollow $(\|)$ & Raman $(\perp)$ \\
$\mathrm{F}+\mathrm{B}$ & FWM $(\perp)$ & Mollow $(\|)$ & FWM $(\|)$ \\
\hline \hline
\end{tabular}

in detail below, we are able to produce lasing with cold atoms as gain medium using three different gain mechanisms: Mollow gain, Raman gain and Four Wave Mixing (FWM). We can control the different mechanisms by the

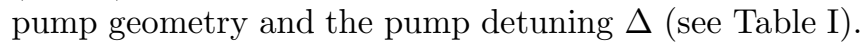
Mollow and Raman gain mechanisms only require a single pump beam $(\mathrm{F})$, whereas FWM only occurs when both pump beams $\mathrm{F}$ and $\mathrm{B}$ are present and carefully aligned. With a single pump beam, we find Mollow gain to be dominating close to the atomic resonance, whereas Raman gain is more important for detunings larger than $|\Delta| \approx 4 \Gamma$. Furthermore, the different gain mechanisms lead to distinct polarizations. Mollow gain generates a lasing mode with a polarization parallel to the pump polarization, because the Mollow amplification is maximum for a field aligned with the driven atomic dipole [8]. On the contrary, different polarizations between the pumping and the amplified waves are necessary to induce a Raman transition between two Zeeman substates: the polarization of the Raman laser is thus orthogonal to the pump polarization. Lastly, the FWM laser has a more complex polarization behaviour, as it is orthogonal for red-, and parallel for blue-detuned pumps. We have checked that for any pump detuning or probe power, the weakprobe FWM reflectivity is stronger for orthogonal probe polarization, as expected from previous experiments and models [14]. We speculate that pump-induced mechanical effects [15] or more complex collective coupling between the atoms and the cavity [16] might be the origin of this polarization behavior.

In Fig. 11we show spatial (transverse) patterns of these lasers, observed by imaging the beam onto a CCD camera. Without any spatial filtering in the cavity, the different lasers (Mollow, Raman and FWM) yield distinct transverse patterns. In Fig. 1(b) [Fig. 1(c)] we show the transverse pattern obtained with a Mollow (Raman) laser. We note that the Mollow laser typically produces transverse patterns with radial symmetries well described by Laguerre-Gauss modes, whereas the modes of the Raman laser are rather Hermite-Gauss modes. The origin of such radial or Cartesian symmetry may arise from the different polarization of those two lasers: the radial symmetry is preserved for the Mollow laser polarization and is broken for the Raman laser one, probably due to slightly different losses in the cavity. Fig. 1(d) shows the transverse pattern of the FWM laser. As phase conjugation mechanisms are at work in such a laser, any transverse

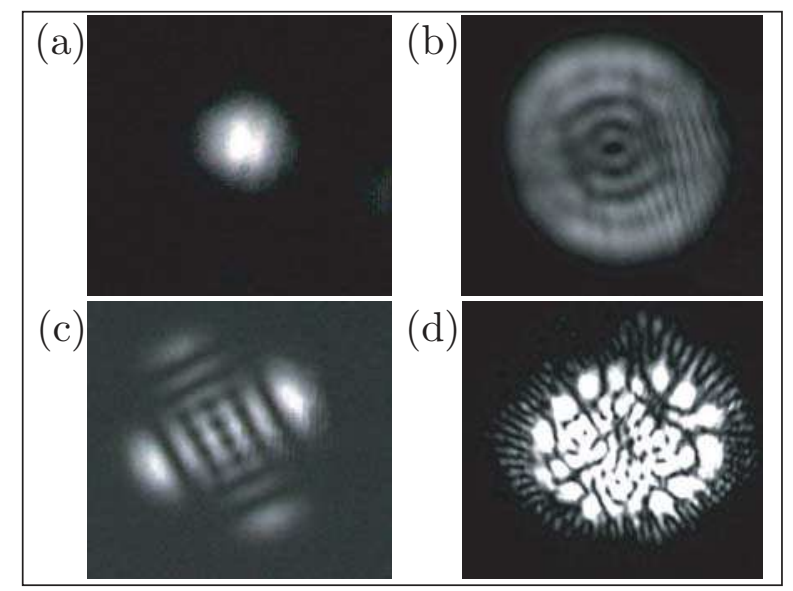

FIG. 1: Transverse modes of cold-atom lasers. (a) Gaussian $\mathrm{TEM}_{00}$ mode, obtained by inserting a small diaphragm in the cavity. Typical modes of (b) the Mollow laser, (c) the Raman laser, and (d) the four-wave mixing laser.

mode can easily cross the lasing threshold and complex lasing patterns are produced [17].

We now turn to a more detailed description of the gain mechanisms of the different lasers. The quantitative understanding of their behavior needs to take into account effects such as pump geometry and parameters (intensity, detuning), gain spectra, gain saturation and mechanical effects induced by the pump beam(s).

Let us first discuss the Mollow laser. Amplification of a weak probe beam can happen when a two-level atom is excited by one strong pump beam $[8,9]$. The corresponding single-pass gain is $g_{\mathrm{M}}=\exp \left[-b_{0} f_{\mathrm{M}}(\Omega, \Delta, \delta)\right]$, where $b_{0}$ is the on-resonance optical thickness (without pump) of the cold-atom cloud. The expression of $f_{\mathrm{M}}(\Omega, \Delta, \delta)$ can be obtained from Optical Bloch Equations [8]:

$$
\begin{aligned}
& f_{\mathrm{M}}(\Omega, \Delta, \delta)=\frac{\Gamma}{2} \frac{|z|^{2}}{|z|^{2}+\Omega^{2} / 2} \times \\
& \quad \operatorname{Re}\left[\frac{(\Gamma+i \delta)(z+i \delta)-i \Omega^{2} \delta /(2 z)}{(\Gamma+i \delta)(z+i \delta)\left(z^{*}+i \delta\right)+\Omega^{2}(\Gamma / 2+i \delta)}\right],
\end{aligned}
$$

where $z=\Gamma / 2-i \Delta$ and $\Omega$ is the Rabi frequency of the atom-pump coupling, related to the pump intensity $I$ by $\Omega^{2}=\mathcal{C}^{2} \Gamma^{2} I /\left(2 I_{\text {sat }}\right)\left(I_{\text {sat }}=1.6 \mathrm{~mW} / \mathrm{cm}^{2}\right.$ is the saturation intensity and $\mathcal{C}$ is the averaged Clebsch-Gordan coefficient of the $F=3 \rightarrow F^{\prime}=4$ transition for a linear polarization). In our setup we observe single-pass gain higher than $50 \%$, with a large gain curve (width $>\Gamma$ ). The shape of the transmission spectrum (inset of Fig. 2) is consistent with Eq. (11). From Eq. (11) we can also predict the maximum gain in respect to the pump parameters $\Omega, \Delta$. We observe good agreement between the behavior of the laser power and of the function $f_{\mathrm{M}}$ when varying $\Delta$ : the maximum gain and laser power are achieved for $|\Delta| \sim 2 \Gamma$ (the exact value depends on $\Omega$ ) and $\Delta=0$ is a local minimum. However, we measured a 


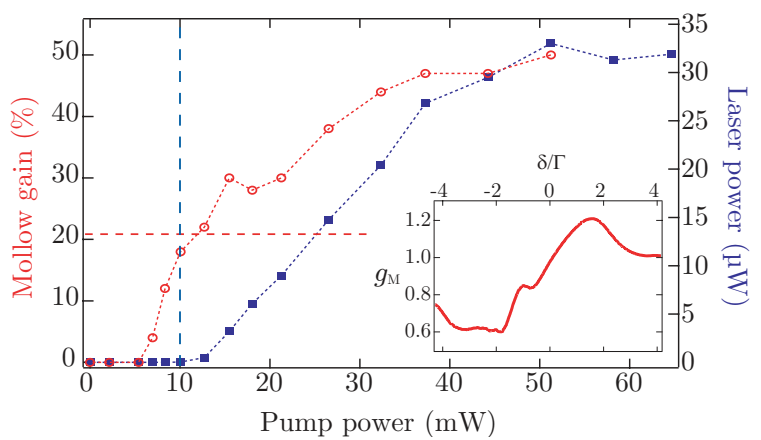

FIG. 2: Laser power (squares) and Mollow gain (open circles) versus pump power, with $b_{0}=11$ and $\Delta=+\Gamma$. Lasing threshold (vertical dashed line) is expected to appear with a gain of about $21 \%$ (horizontal dashed line), in good agreement with the experimental data. Inset: Typical weak-probe transmission spectrum.

lower maximum gain than predicted by Eq. (11). This is due to gain-saturation induced by re-scattering of spontaneous emission inside the atomic cloud [18].

As shown in Fig. 2 (squares), we observe a Mollow laser emission with an output intensity reaching $35 \mu \mathrm{W}$. Taking into account the round-trip losses $\mathcal{L}$, the condition for laser oscillation is $g_{\mathrm{M}}^{2}(1-\mathcal{L})>1$. This corresponds to a gain at threshold of $g_{\mathrm{M}}=1.21$ (horizontal line in Fig. 2), in good agreement with the observation.

When the pump frequency in detuned farther away from the atomic resonance, Raman gain becomes dominant. Raman gain relies on the pump-induced population inversion among the different light-shifted $m_{F}$ Zeeman sublevels of the $F=3$ hyperfine level [10, 19]. Single-pass Raman gain of a weak probe can be written $g_{\mathrm{R}}=e^{-b_{0} f_{\mathrm{R}}(\Omega, \Delta, \delta)}$. For $|\Delta| \gg \Gamma, f_{\mathrm{R}}(\Omega, \Delta, \delta)$ is given by

$$
f_{\mathrm{R}}=-\frac{\Omega^{2}}{\Delta^{2}}\left(\frac{A_{1}}{\left(\delta+\delta_{R}\right)^{2}+\gamma^{2} / 4}-\frac{A_{2}}{\left(\delta-\delta_{R}\right)^{2}+\gamma^{2} / 4}\right)
$$

where $A_{1,2}$ are the respective weights of the amplification and absorption, $\delta_{\mathrm{R}}$ is the frequency difference between the Zeeman sublevels and $\gamma$ is the width of the Raman resonance [19]. We have observed the laser spectrum with a beat-note experiment, and we have checked that its frequency corresponds to the maximum gain and is related to the differential pump-induced light-shift $\delta_{\mathrm{R}}$ of the different Zeeman sublevels. The width of the Raman resonance $\gamma$ is related to the elastic scattering rate of the pump photons and is much lower than $\Gamma$, due to the strong detuning $\Delta$. The result is thus a much narrower gain spectrum than in the previous case (inset of Fig. 3). This leads to an important practical limitation of the single-pumped Raman laser: atoms are pushed by the pump beam, acquiring a velocity $v$, and the subsequent Doppler shift becomes quickly larger than the width of the gain spectrum. As a consequence, the gain in the

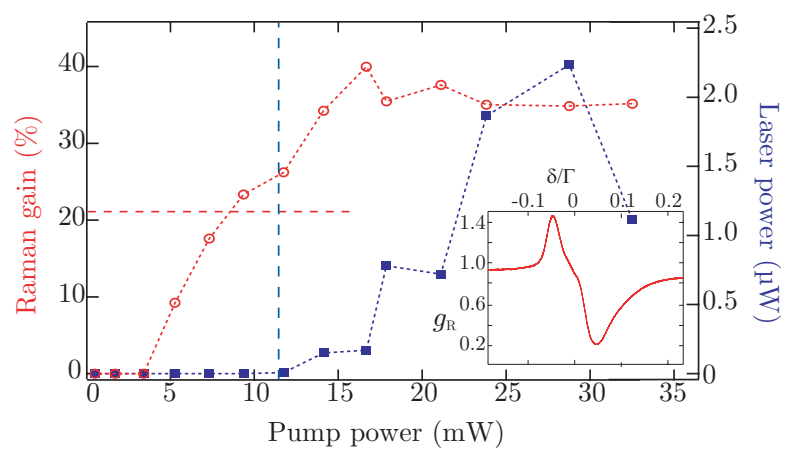

FIG. 3: Laser power (squares) and Raman gain (open circles) versus pump power, with $b_{0}=10$ and $\Delta=-7 \Gamma$. Lasing threshold (vertical dashed line) is expected to appear with a gain of about $21 \%$ (horizontal dashed line), in good agreement with the experimental data. Inset: Typical weak-probe transmission spectrum.

cold-atom cloud is no longer the same for a wave copropagating with the pump beam $(\mathrm{F})$ and the wave running in the counterpropagating direction. For the copropagating direction, the relative Doppler shift is negligible, whereas for the counterpropagating wave, a Doppler shift of $\sim 2 \omega_{\mathrm{A}} v / c$, larger than the width of the gain spectrum, leads to a suppression of the corresponding gain. As a consequence, emission of our Raman laser stops after $\approx 20 \mu$ s [20].

In Fig. 3 we plot the output power of the Raman laser as a function of pump power. A comparison with the single-pass gain $g_{R}$ is again in good agreement for the threshold condition $g_{\mathrm{R}}^{2}(1-\mathcal{L})>1$ : for Raman gain above $21 \%$ laser emission occurs. As shown in Fig. 3 (squares), the output power of the Raman laser emission $(\approx 2 \mu \mathrm{W})$ is much lower than the Mollow laser one. This lower output power might arise from a lower saturation intensity for Raman gain 21]. Nevertheless, with a weak signal, the Raman gain can be as high as $g_{\mathrm{R}}=2[21]$.

We have observed another lasing mechanism when a balanced pumping scheme using two counterpropagating pump beams $\mathrm{F}$ and $\mathrm{B}$ is used. In this configuration FWM appears [12, 13]. The creation of photons in a reflected wave, resulting from a phase conjugation process, can also be considered as a gain mechanism. This is reminiscent of optical parametric oscillation where signal and idler photons are created under a phase matching condition. In the inset of Fig. 4 we show the FWM signal $R_{c}$ (expressed as the reflection normalized to the incident probe power) illustrating the narrow spectrum of this phase conjugation signal. As expected, the maximum gain corresponds to the degenerate case $\delta=0[14]$. Thanks to constructive interference between transmitted and reflected waves, this mechanism produces huge double-pass gain with cold atoms 21] and it is thus an efficient mechanism to trigger laser oscillations 22]. Due to these interference effects, the threshold for laser oscil- 


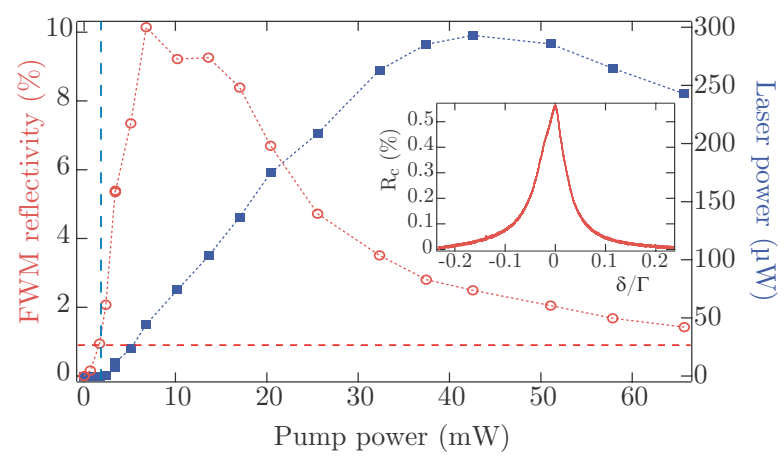

FIG. 4: Laser power (squares) and phase-conjugate reflectivity due to four-wave mixing (open circles) versus pump power, with $b_{0}=10$ and $\Delta=-8 \Gamma$. Lasing threshold (vertical dashed line) is expected for a reflectivity around 1\% (horizontal dashed line), in good agreement with the experimental data. Inset: Example of a weak-probe reflectivity spectrum.

lation is very different from the previous cases [21, 22], and is given by

$$
R_{c}>[(1-\sqrt{\tilde{R}}) /(1+\sqrt{\tilde{R}})]^{2}=0.9 \%
$$

where $\tilde{\mathcal{R}}=1-\mathcal{L}$. This criterion (horizontal line in Fig. (4) is well respected for the threshold of our laser. The output power of this laser is quite strong $(300 \mu \mathrm{W})$, with an energy conversion efficiency of $0.75 \%$ in this case. As two pump beams are used in this situation, the mechanical effects based on radiation pressure will be negligible and lasing can be sustained for a long time. However dipole forces can induce atomic bunching, and change the effective pump intensity interacting with the atoms [15].

In conclusion, we presented in this Letter three types of laser using a sample of cold atoms as gain medium. Three different gain mechanisms were demonstrated as being efficient enough to allow lasing, even with a low finesse cavity. Comparison between Mollow and Raman laser shows that the latter has a significantly lower power, although their gain are of the same order of magnitude. These two mechanisms can produce high gain at frequencies slightly detuned from the pump, allowing to distinguish between stimulated photons from the laser mode and scattered photons from the pump beam. Thus, they seem to be good candidates for the search of random lasing in cold atoms, and the combination of these gains with multiple scattering will be the subject of further investigations. In addition, the ability to continuously tune from a Mollow to a Raman laser (by changing the pump detuning), may allow to study the transformation of transverse patterns from Laguerre-Gauss to Hermite-Gauss modes [23]. The
FWM laser is the most efficient in terms of power, and it should be possible to study its noise spectrum down to the shot noise level. This laser has many analogies to an optical parametric oscillator and seems to be a good candidate to explore non classical features of light, such as the production of twin beams 24, 25]. Lastly, the coupling between the cavity mode and the atomic internal and external degrees of freedom, may also reveal interesting dynamics, especially if a high-finesse cavity is used [16, 26, 27].

The authors thank G.-L. Gattobigio for his help at the early stages of the experiment. This work is supported by INTERCAN, DGA and ANR-06-BLAN-0096.

* Electronic address: Robin.Kaiser@inln.cnrs.fr

[1] V. S. Letokhov, Sov. Phys. JETP 26, 835 (1968).

[2] D. S. Wiersma, Nature Physics 4, 359 (2008).

[3] G. Labeyrie et al., Phys. Rev. Lett. 83, 5266 (1999).

[4] G. Labeyrie et al., Phys. Rev. Lett. 91, 223904 (2003).

[5] T. Wang et al., Phys. Rev. A 75, 033802 (2007); J. O. Day, E. Brekke and T. G. Walker, Phys. Rev. A 77, 052712 (2008).

[6] M. S. Malcuit et al., Phys. Rev. Lett. 59, 1189 (1987).

[7] R. E. Slusher et al., Phys. Rev. Lett. 55, 2409 (1985).

[8] B. R. Mollow, Phys. Rev. A 5, 2217 (1972).

[9] F. Y. Wu et al., Phys. Rev. Lett. 38, 1077 (1977).

[10] D. Grison et al., Europhys. Lett. 15, 149 (1991); J. W. R. Tabosa et al., Phys. Rev. Lett. 66, 3245 (1991).

[11] L. Hilico, C. Fabre, and E. Giacobino, Europhys. Lett. 18, 685 (1992).

[12] A. Yariv and D. M. Pepper, Opt. Lett. 1, 16 (1977).

[13] R. L. Abrams and R. C. Lind, Opt. Lett. 2, 94 (1978), Opt. Lett. 3, 205 (1978).

[14] A. Lezama, G. C. Cardoso and J. W. R. Tabosa, Phys. Rev. A 63, 013805 (2000).

[15] G.-L. Gattobigio et al., Phys. Rev. A 74, 043407 (2006).

[16] D. Nagy et al., Europhys. Lett. 74, 254 (2006).

[17] R. C. Lind and D. G. Steel, Opt. Lett. 6, 554 (1981).

[18] L. Khaykovich, N. Friedman and N. Davidson, Eur. Phys. J. D 7, 467 (1999).

[19] T. M. Brzozowski et al., Phys. Rev. A 71, 013401 (2005).

[20] At time scale larger than $100 \mu \mathrm{s}$, the single-pumped Mollow laser suffers also from mechanical effects of the pump.

[21] F. Michaud et al., J. Opt. Soc. Am. B 24, A40 (2007).

[22] M. Pinard, D. Grandclement, and G. Grynberg, Europhys. Lett. 2, 755 (1986).

[23] E. G. Abramochkin and V. G. Volostnikov, J. Opt. A: Pure Appl. Opt. 6, S157 (2004).

[24] M. Vallet, M. Pinard, and G. Grynberg, Europhys. Lett. 11, 739 (1990).

[25] C. F. McCormick et al., Opt. Lett. 32, 178 (2007).

[26] D. Kruse et al., Phys. Rev. Lett. 91, 183601 (2003).

[27] R. Miller et al., J. Phys. B 38, S551 (2005). 